\title{
The Euler Characteristic is the Unique \\ Locally Determined Numerical Homotopy Invariant of Finite Complexes
}

\author{
Norman Levitt \\ Department of Mathematics, Rutgers University, \\ New Brunswick, NJ 08903, USA
}

\begin{abstract}
If a numerical homotopy invariant of finite simplicial complexes has a local formula, then, up to multiplication by an obvious constant, the invariant is the Euler characteristic. Moreover, the Euler characteristic itself has a unique local formula.
\end{abstract}

\section{Introduction}

The Euler characteristic $\chi$ is the best known as well as the most ancient topological invariant. For a finite simplicial complex $K$ (or, more generally, a $\mathrm{C}-\mathrm{W}$ complex) there is the familiar definition

$$
\chi(K)=\sum_{i=0}^{\operatorname{dim} K}(-1)^{i} c_{i}
$$

where $c_{i}=$ number of $i$-simplices (or $i$-cells if $K$ is a $\mathrm{C}$-W complex.) That $\chi(K)$ is an invariant of homotopy type follows from the alternative definition

$$
\chi(K)=\sum_{i=0}^{\operatorname{dim} K}(-1)^{i} \operatorname{rank} H_{i}(K ; \mathbb{Z})
$$

It is well known and easily verified that $\chi(K)$ is locally determined in the sense that given $K$, we may assign to each vertex $v \in K$ a rational number $e_{1}(v)$ such that $\chi(V)=\sum_{v} e_{1}(v)$. Here, $e_{1}(v)$ depends only on the simplicial structure of star 
$v=\bigcup_{v \in \sigma} \sigma(\sigma$ a simplex of $K)$ and is given by

$$
e_{1}(v)=\sum_{i} \frac{(-1)^{i}}{i+1} \cdot s_{i}(v)
$$

where $s_{i}(v)$ is the number of $i$-simplices of $K$ containing $v$. Since star $v$ is, simplicially, the cone $c($ link $v)$, we may think of $e_{1}(v)$ as an invariant of the simplicial isomorphism type of link $v=\bigcup_{v \notin \sigma \subset s t a r v} \sigma$, i.e.,

$$
e_{1}(v)=e(\text { link } v)=1+\sum_{i=0}^{\operatorname{dimlink} v} \frac{(-1)^{i+1}}{i+2} \cdot(\text { number of } i \text {-simplices of link } v) \text {. }
$$

Of course, there are countless other $\mathbb{Z}$-valued (or $\mathbb{R}$-valued) invariants of finite complexes of finite complexes. It seems natural to ask whether any of these, other than the Euler characteristic, is locally determined in this sense. Specifically, let $\rho$ denote any $\mathbb{R}$-valued homotopy invariant of finite complexes. We always assume, by way of normalization, that $\rho(\varnothing)=0$. Consider a real-valued function $d(L)$ defined on the set of finite simplicial complexes and depending only on the simplicial isomorphism type of $L$. We say that $\rho$ is locally determined by $d$ if and only if given any finite simplicial complex $K$ we have $\rho(K)=\sum_{v \in K} d($ link $v)$, where the sum is taken over the vertices $v$ of $K$. Clearly, the example we have in mind is the Euler characteristic $\chi$, locally determined by $e$ as above, and our question is whether there are any other numerical homotopy invariants (in a nontrivial sense) which are locally determined. The answer turns out to be negative.

Theorem A. Let $\rho$ be any $\mathbb{R}$-valued homotopy invariant of finite complexes locally determined by some function d on simplicial-isomorphism classes of finite complexes. Then $\rho=\rho(\mathrm{pt}) \cdot \chi$.

In other words, up to multiplication by a constant, $\chi$ is the unique locally determined homotopy invariant.

We prove Theorem $\mathrm{A}$ in the following form:

Theorem $\mathbf{A}^{\prime}$. If $\rho$ is an $\mathbb{R}$-valued homotopy invariant of finite complexes locally determined by $d$ and such that $\rho(\mathrm{pt})=$.1 , then $\rho \equiv \chi$.

Theorem $\mathrm{A}$ obviously implies Theorem $\mathrm{A}^{\prime}$ and is, in turn, implied by it for the following reason: Let $\rho$ be as in the statement of Theorem A. If $\rho(\mathrm{pt}) \neq$.0 , replace $\rho$ by $\rho^{\prime}=\rho / \rho\left(\right.$ pt.) and apply Theorem $\mathrm{A}^{\prime}$ to conclude $\rho^{\prime}=\chi$, hence $\rho=\rho(\mathrm{pt}$.) $\cdot \chi$. If, however, $\rho\left(\mathrm{pt}\right.$.) $=0$ let $\rho^{\prime}=\rho+\chi$. Applying Theorem $\mathrm{A}^{\prime}$ to $\rho^{\prime}$, we have $\rho^{\prime}=\chi$ hence $\rho=0=\rho($ pt. $) \cdot \chi$.

The author is indebted to the referee for pointing out that the techniques below will, in fact, lead to a somewhat stronger result.

Consider compact PL $n$-manifolds (not necessarily closed). Let $\rho$ now denote a real-valued PL-homeomorphism invariant of such manifolds. Let $d$ be a 
real-valued function defined on triangulations of $S^{n-1}$ and $D^{n-1}$. Then the notion of $\rho$ being locally determined by $d$ transcribes, in an obvious way, to this context from the definition given above. Corresponding to Theorem $\mathbf{A}^{\prime}$ we have

Theorem $\mathbf{A}^{\prime \prime}$. If $\rho$ is an $\mathbb{R}$-valued invariant of compact $P L$ n-manifolds with $\rho(\varnothing)=0, \rho\left(D^{n}\right)=1$ and $\rho$ is locally determined by some function $d$, then $\rho=\chi$.

Note that Theorem $A^{\prime \prime}$ does, in fact, imply Theorem $A^{\prime}$. Let $\rho$ be a numerical homotopy invariant of finite complexes with $\rho(\varnothing)=0, \rho(\mathrm{pt}$. $)=1$. Then, for any $n$, $\rho$ is, a fortiori, a PL-homeomorphism invariant of compact PL $n$-manifolds with $\rho\left(D^{n}\right)=1$. If $\rho$ is locally determined, then Theorem A" tells us that $\rho\left(M^{n}\right)=\chi\left(M^{n}\right)$ for compact PL manifolds $M^{n}$ ( $n$ arbitrary). However, given a finite complex $K$, there exists a compact manifold $M^{n}$ with $K$ homotopically equivalent to $M^{n}$ (see, e.g., [W1]). Hence $\rho(K)=\rho\left(M^{n}\right)=\chi\left(M^{n}\right)=\chi(K)$.

The observation above notwithstanding, we shall, in the interest of simplicity of exposition, prove Theorem $\mathrm{A}^{\prime}$ directly first and then show how Theorem $\mathrm{A}^{\prime \prime}$ follows by a straightforward modification of the proof.

If we now go on to ask how many functions $d$, in addition to the $e$ given above, locally determine $\chi$, we find, in fact, that an even greater degree of rigidity prevails than is asserted by Theorem A. Not only is $\chi$ the only locally determined homotopy invariant which evaluates to 1 on a point but, as well, there is only one function, namely $e(L)$, which determines it. We rephrase this:

Theorem B. If $\chi$ is locally determined by $d$, then $d=e$.

Some remarks before we proceed to the proofs: If we examine more restricted classes of finite complexes, Theorem A no longer holds. For instance, if we look at the class of triangulated, oriented closed $4 k$-manifolds $M$, then the signature of $M$, certainly an invariant of orientation-preserving homotopy type within this class of spaces, is locally determined by a function defined on simplicial-isomorphism classes of triangulated, oriented $(4 k-1)$-spheres. This is a special case of the fact that rational Pontrjagin classes (as well as other PL characteristic classes) are locally determined. See $[C],[L],[L R]$, and $[G M]$ for details.

Moreover, as the referee has astutely pointed out, Theorem B fails as well in the context of closed PL $n$-manifolds. Given a combinational triangulation of such a manifold $M$, let $c_{i}$ denote the number of $i$-simplices. Klee $[\mathrm{K}]$ discovered a family of algebraic relations among the $c_{i}$ valid for all $M$ and Wall [W2] showed this list to be exhaustive. In consequence, there are nonstandard formulae for $\chi(M)$ in terms of $c_{i}$ differing from the classical formula cited in the first paragraph. For example (and, once more, the author is indebted to the referee for the observation),

$$
\chi\left(M^{n}\right)=c_{0}+\sum_{j=1}^{[(n+1) / 2]}(-1)^{j} 2 B_{2 j} c_{2 j-1}
$$


where $B_{2 j}$ is the $2 j$ th Bernoulli number. From this it immediately follows that if we set

$$
\left.d(L)=1+\sum_{j=0}^{[(n-1) / 2]} \frac{(-1)^{j+1}}{j+1} B_{2 j+2} \text { (number of } 2 j \text { simplices of } L\right)
$$

then $d$ locally determines $\chi$ on closed $n$-manifolds.

A word concerning notation: We abbreviate star $v$ by st $v$ and link $v$ by $1 \mathrm{k} v$. If there is any ambiguity as to which ambient complex $K$ is under consideration, we resolve this by recourse to subscripts, e.g. $\mathrm{lk}_{\mathrm{K}} v, \mathrm{st}_{\mathrm{K}} v$, etc.

Finally, we note that our proof applies to $\mathbb{C}$-valued or $\mathbb{Q}$-valued invariants as well.

\section{Proof of Theorem $\mathbf{A}^{\prime}$}

Theorem $A^{\prime}$ follows immediately from:

Lemma 1. Let $\rho$ be a numerical homotopy invariant of finite complexes locally determined by some function $d$. Let $K$ be a finite simplicial complex with $K=$ $K_{0} \cup K_{1}, K_{0} \cap K_{1}=K_{2}$ where $K_{0}, K_{1}, K_{2}$ are subcomplexes of $K$. Then $\rho(K)=$ $\rho\left(K_{0}\right)+\rho\left(K_{1}\right)-\rho\left(K_{2}\right)$.

The derivation of Theorem $A^{\prime}$ from Lemma 1 comes via a straightforward induction. Given Lemma 1 and the hypothesis that $\rho(\mathrm{pt})=$.1 , it is immediate that for a 0-complex (i.e., discrete finite set) $K, \rho(K)=$ number of points of $K=\chi(K)$. So assume, inductively, that $\rho=\chi$ holds for complexes of dimension $\leq k$ and for $(k+1)$-complexes having $\leq j(k+1)$-simplices. Let $K$ be a $(k+1)$-dimensional complex with exactly $(j+1)(k+1)$-simplices. Choose a $(k+1)$-simplex $\sigma$. Let $K_{0}=\sigma, K_{1}=K$-int $\sigma$, so that $K_{2}=K_{0} \cap K_{1}$ is a $k$-sphere. Then

$$
\rho(K)=\rho\left(K_{0}\right)+\rho\left(K_{1}\right)-\rho\left(K_{2}\right)=1+\chi\left(K_{1}\right)-\chi\left(S^{k}\right)=\chi\left(K_{1}\right)+(-1)^{k+1}=\chi(K)
$$

To prove Lemma 1 , in turn, it is technically convenient to consider finite regular cell complexes in addition to the more special category of finite simplicial complexes. (See [SCF] for definitions and basic properties of regular cell complexes.) Let $d_{1}$ be a function defined on pairs $(J, p)$ where $J$ is a regular cell complex which is the union of cells all containing the vertex $p$. It is understood that $d_{1}$ depends only on the isomorphism class of $(J, p)$ as a regular cell-complex pair. Thus in the case when $J$ happens to be simplicial, we see that $d_{1}$ depends only on the simplicial isomorphism class of $(J, p)$ and thus, since $J$ will in this instance be $c\left(\mathrm{k} p\right.$ ), only on the simplicial isomorphism class of $\mathrm{lk} p$. Consequently, $d_{1}$ may be viewed as an extension of a function $d(L)$, defined on simplicial complexes of the sort we have heretofore been considering (that is, $d(L)=d_{1}(c L, *)$ ). We say that $d_{1}$ determines an $\mathbb{R}$-valued homotopy invariant $\rho$ of finite regular cell-complexes 
if and only if $\rho(K)=\sum_{v} d_{1}(s t v, v)$ where the sum is taken over the vertices $v$ of $K$ and where st(v) is now understood to mean the union of all those cells of $K$ containing $v$.

Lemma 2. If $d$ (defined on simplicial complexes) locally determines $\rho$ on simplicial complexes, then there is an extension of $d$ to regular cell-complex pairs $(J, p)$ as above which locally determines $\rho$ on regular cell-complexes.

(Of course, it is understood that since regular cell-complexes are triangulable - in fact the first barycentric subdivision is a simplicial complex-the invariant $\rho$ automatically extends to regular cell-complexes.)

The proof of Lemma 2 is quite straightforward. Given $(J, p)$ let $K$ be the first barycentric subdivision of $J$, and hence a simplicial complex. Define $d_{1}(J, p)$ as follows: let $e$ be a cell of $J, b_{e}$ its barycenter, hence a vertex of $K$. Let $V(e)$ denote the number of vertices of the regular cell $e$. Then set $d_{1}(J, p)=\sum_{e}(1 / v(e)) d\left(1 \mathrm{k}_{K} b_{e}\right)$ where the sum is taken over all cells of $J$. The assertion that $d_{1}$ must locally determine $\rho$ on regular cell complexes is an immediate consequence of this definition.

We now proceed to the proof of Lemma 1 . Assume that $d$, which locally determines $\rho$ on simplicial complexes, has been extended to $d_{1}$, which locally determines $\rho$ on regular cell-complexes. Let $M$ be a finite regular cell-complex and let $I$ denote, as usual, the unit interval as a simplicial complex with one 1 -simplex $[0,1]$ and two vertices 0 and $1 . M \times I$ is then well defined as a regular cell-complex without need of further subdivision. If $M$ has vertices $v_{1}, \ldots, v_{k}$, then $M \times I$ has vertices $u_{1}, \ldots, u_{k}, w_{1}, \ldots, w_{k}$ where $u_{i}=\left(v_{i}, 0\right), w_{i}=\left(v_{i}, 1\right)$ :

\section{Lemma 3.}

$$
\begin{aligned}
\sum_{i=1}^{k} d_{1}\left(\mathrm{st}_{M \times I} u_{i}, u_{i}\right) & =\sum_{i=1}^{k} d_{1}\left(\mathrm{st}_{M \times I} w_{i}, w_{i}\right) \\
& =\frac{1}{2} \sum_{i=1}^{k} d_{1}\left(\mathrm{st}_{M} v_{i}, v_{i}\right)=\frac{1}{2} \rho(M)
\end{aligned}
$$

Proof. $\left(\mathrm{st}_{M \times I} u_{i}, u_{i}\right)$ is isomorphic as a regular cell-complex pair to (st s. $\left._{M I} w_{i}, w_{i}\right)$, thus it is immediate that

$$
\sum_{i=1}^{k} d_{1}\left(\mathrm{st}_{M \times I} u_{i}, u_{i}\right)=\sum_{i=1}^{k} d_{1}\left(\mathrm{st}_{M \times I} w_{i}, w_{i}\right)
$$

But

$$
\sum_{i=1}^{k} d_{1}\left(\mathrm{st}_{M \times I} u_{i}, u_{i}\right)+\sum_{i=1}^{k} d_{1}\left(\mathrm{st}_{M \times I} w_{i}, w_{i}\right)=\rho(M \times I)=\rho(M)=\sum_{i=1}^{k} d_{1}\left(\mathrm{st}_{M} v_{i}, v_{i}\right)
$$

which yields the remainder of the lemma. 
Now let $I^{\prime}$ denote the first subdivision of $I$ with two 1 -simplices $\left[0, \frac{1}{2}\right]$ and $\left[\frac{1}{2}, 1\right]$ and three vertices $0, \frac{1}{2}, 1$. With $M, v_{i}$ as above, $M \times I^{\prime}$ is a regular cell-complex with vertices $u_{i}=\left(v_{i}, 0\right), w_{i}=\left(v_{i}, 1\right), x_{i}=\left(v_{i}, \frac{1}{2}\right)$. Clearly, $\left(\mathrm{st}_{M \times I^{\prime}} u_{i}, u_{i}\right)$ is isomorphic as a regular cell-complex pair to $\left(\mathrm{st}_{M \times I} u_{i}, u_{i}\right)$ and similarly for $w_{i}$. This observation leads to:

Lemma 4. $\sum_{i=1}^{k} d_{1}\left(\mathrm{st}_{M \times I^{\prime}} x_{i}, x_{i}\right)=0$.

Proof.

$$
\rho\left(M \times I^{\prime}\right)=\sum_{i=1}^{k} d_{1}\left(\mathrm{st}_{M \times I^{\prime}} u_{i}, u_{i}\right)+\sum_{i=1}^{k} d_{1}\left(\mathrm{st}_{M \times I^{\prime}} w_{i}, w_{i}\right)+\sum_{i=1}^{k} d_{1}\left(\mathrm{st}_{M \times I^{\prime}} x_{i}, x_{i}\right) .
$$

Also

$$
\rho\left(M \times I^{\prime}\right)=\rho(M \times I)=\sum_{i=1}^{k} d_{1}\left(\mathrm{st}_{M \times I} u_{i}, u_{i}\right)+\sum_{i=1}^{k} d_{1}\left(\mathrm{st}_{M \times I} w_{i}, w_{i}\right) .
$$

By the remarks immediately preceding the statement of the lemma, the two summands on the right-hand side of the second equation are respectively equal to the first two summands in the right-hand side of the second. Hence the remaining summand, namely $\sum_{i=1}^{k} d_{1}\left(\mathrm{st}_{M \times I^{\prime}} x_{i}, x_{i}\right)$, must vanish.

Now we complete the proof of Lemma 1. Let $K=K_{0} \cup K_{1}$ be a simplicial complex with $K_{0} \cap K_{1}=K_{2}$. We construct a homotopy-equivalent regular cellcomplex $K_{0} \cup\left(K_{2} \times I^{\prime}\right) \cup K_{1}=B$ where $K_{0}, K_{1}$ are now disjoint and $K_{2} \times 0$ is identified with the copy of $K_{2}$ in $K_{0}$ and $K_{2} \times 1$ with the copy of $K_{2}$ in $K_{1}$. Let $B_{0}=K_{0} \cup\left(K_{2} \times\left[0, \frac{1}{2}\right]\right), B_{1}=K_{2} \times\left[\frac{1}{2}, 1\right] \cup K_{1}$. Thus $B_{0} \cap B_{1}=K_{2} \times \frac{1}{2}$. We denote the vertices of $K_{2}$ by $v_{1}, \ldots, v_{k}$. Thus $K_{2} \times \frac{1}{2}$ has vertices $x_{i}=\left(v_{i}, \frac{1}{2}\right), i=$ $1, \ldots, k$. Two elementary observations:

(i) ( $\left(\mathrm{st}_{B_{1}} x_{i}, x_{i}\right)$ is isomorphic (as a regular cell-complex pair) to $\left(\mathrm{st}_{K_{2} \times I}\left(v_{i}, 0\right)\right.$, $\left.\left(v_{i}, 0\right)\right)$ and likewise for $\left(\mathrm{st}_{\boldsymbol{B}_{2}} x_{i}, x_{i}\right)$.

(ii) $\left(\mathrm{st}_{B}, x_{i}, x_{i}\right)$ is identical with (st $\mathrm{K}_{2} \times \gamma_{i}, x_{i}, x_{i}$ ).

Now, since $d_{1}$ locally determines $\rho$, we have

$$
\rho\left(K_{0}\right)=\rho\left(B_{0}\right)=\sum_{i=1}^{k} d_{1}\left(\mathrm{st}_{B_{0}} x_{i}, x_{i}\right)+Y,
$$

where $Y$ involves only the stars of vertices of $B_{0}$ not in $K_{2} \times \frac{1}{2}$. Likewise,

$$
\rho\left(K_{1}\right)=\sum_{i=1}^{k} d_{1}\left(\mathrm{st}_{B_{1}} x_{i}, x_{i}\right)+Z
$$


where $Z$ involves only the stars of vertices of $B_{1}$ not in $K_{2} \times \frac{1}{2}$. Thus, by Lemma 3 and observation (i) above, we see immediately that

$$
\sum_{i=1}^{k} d_{1}\left(\mathrm{st}_{B_{0}} x_{i}, x_{i}\right)=\sum_{i=1}^{k} d_{1}\left(\mathrm{st}_{B_{1}} x_{i}, x_{i}\right)=\frac{1}{2} \rho\left(K_{2}\right) .
$$

On the other hand, it is directly seen that $\rho(K)=\rho(B)=\sum_{i=1}^{k} d_{1}\left(\mathrm{st}_{B_{1}}, x_{i}, x_{i}\right)+$ $Y+Z$. However, $\sum_{i=1}^{k} d_{1}\left(\mathrm{st}_{B} x_{i}, x_{i}\right)$ vanishes by virtue of observation (ii) and Lemma 4. So $\rho(K)=Y+Z=\left[\rho\left(K_{0}\right)-\frac{1}{2} \rho\left(K_{2}\right)\right]+\left[\rho\left(K_{1}\right)-\frac{1}{2} \rho\left(K_{2}\right)\right]=\rho\left(K_{0}\right)+$ $\rho\left(K_{1}\right)-\rho\left(K_{2}\right)$. The proof of Lemma 1, and hence of Theorems $A$ and $\mathrm{A}^{\prime}$, is thus complete.

The kindred result Theorem $\mathrm{A}^{\prime \prime}$ is easily established by a slightly modified version of this reasoning. The key point is the following lemma, analogous to Lemma 1.

Lemma 5. Let $\rho$ be a locally determined numerical PL-homeomorphism invariant for compact $P L$-manifolds. Let $K$ be a compact $P L n$-manifold of the form $K=K_{0} \cup K_{1}$ where $K_{0}, K_{1}$ are themselves compact n-manifolds and where $K_{2}=$ $K_{0} \cap K_{1}$ is a codimension 0 submanifold of both $\partial K_{0}$ and $\partial K_{1}$. Then $\rho(K)=\rho\left(K_{0}\right)+$ $\rho\left(K_{1}\right)-\rho\left(K_{2} \times I\right)$.

First, we see quite easily that Lemma 5 implies Theorem $A^{\prime \prime}$. For a compact PL $n$-manifold $M$, let $h(M)$ denote the dimension of the highest dimensional handle in a handlebody-decomposition of $M$ where this highest dimension is minimal (with respect to all possible handlebody structures). Our proof runs by induction on $h(M)$.

If $h(M)=0$, then $M$ is the disjoint union of some finite number $m$ of $n$-disks, whence $\rho(M)=m=\chi(M)$.

Now suppose $\mathrm{A}^{\prime \prime}$ holds for all compact manifolds $M$ with $h(M) \leq j<n$. Consider $M_{1}$ with $h\left(M_{1}\right)=j+1$. Then $M_{1}=M \cup((j+1)$-handles $)$ where $h(M) \leq j$, whence $\rho(M)=\chi(M)$ by inductive assumption. Let $N$ denote the union of all the $(j+1)$-handles of $M_{1}$, i.e., if there are $m$ such handles, $N$ is the disjoint union of $m n$-disks, and so $\rho(N)=m=\chi(N)$. Let $L=M \cap N \subseteq \partial M, \partial N$. $L$ is the disjoint union of $m$ copies of $S^{j} \times D^{n-j-1}$, hence $h(L \times I)=j$ and $\rho(L \times I)=$ $\chi(L \times I)=m\left(1+(-1)^{j}\right)$. By lemma 5 ,

$$
\begin{aligned}
\rho\left(M_{1}\right) & =\rho(M)+\rho(N)-\rho(L \times I)=\chi(M)+\chi(N)-\chi(L \times I) \\
& =\chi(M)+\chi(N)-\chi(L)=\chi\left(M_{1}\right) .
\end{aligned}
$$

The induction is thus complete.

As for Lemma 5 itself, we note that the argument for Lemma 1 goes through almost word for word. Note that lemma 2 holds in the context of regular cell-complex decompositions of PL manifolds. The analogue of Lemma 3 holds where $M$ is now a compact triangulated $(n-1)$-manifold. The modified result 
reads

$$
\sum_{i=1}^{k} d_{1}\left(\mathrm{st}_{M \times I} u_{i}, u_{i}\right)=\sum_{i=1}^{k} d_{1}\left(\mathrm{st}_{M \times I} w_{i}, w_{i}\right)=\frac{1}{2} \rho(M \times I)
$$

Lemma 4 holds as well in this context.

The computations leading to Lemma 1 now serve equally well for Lemma 5 . Here it need only be observed that if $K$ is a compact PL $n$-manifold decomposed as $K_{0} \cup K_{1}$ (with $K_{2}=K_{0} \cap K_{1}$ a codimension-0 submanifold of $\partial K_{0}$ and $\partial K_{1}$ ), then $B$ (as defined in the proof of Lemma 1 ) is now a compact PL $n$-manifold PL homeomorphic to $K$ while $B_{0}, B_{1}$ are PL manifolds homeomorphic, respectively, to $K_{0}$ and $K_{1}$. The proof then goes through substituting $\rho\left(K_{2} \times I\right)$ for $\rho\left(K_{2}\right)$ as appropriate.

\section{Proof of Theorem B}

As noted in the introduction, the Euler characteristic $\chi$ is locally determined by

$$
e(L)=1+\sum_{i=0}^{\operatorname{dim} L} \frac{(-1)^{i+1}}{i+2}(\text { number of } i \text {-simplices of } L) .
$$

We now show that no other function on simplicial isomorphism classes of finite complexes can locally determine $\chi$.

To this end, let $d$ be some other function for which $\chi(K)=\sum_{v} d(\mathrm{lk} V)$ for all finite simplicial complexes $K$. Given any simplicial complex $J$ with $\sigma$ a simplex, we call $\sigma$ a maximal simplex of $J$ if and only if $\sigma$ is not a face of any larger simplex. Note that any finite complex is the union of its maximal simplices.

Our proof that $d(L) \equiv e(L)$ proceeds via induction on the number of maximal simplices of $L$. In the case where $L$ has but one maximal simplex, it is clear that $L$ must be isomorphic to a standard simplex, say $\Delta^{k}$ for some $k \geq 0$. Consider $K=v * L$ for some disjoint vertex $v$. $v * L$ is of course isomorphic to $\Delta^{k+1}$ so $1=\chi(v * L)=d(L)+\sum_{i=0}^{k} d\left(\mathrm{k}_{K} v_{i}\right)$ (where $v_{0}, \ldots, v_{k}$ are the vertices of $L$ ) since $L=\mathrm{lk}_{K} v$. But $\mathrm{lk}_{K} v_{i}$ is obviously a $k$-simplex for $i=0, \ldots, k$. So we have $d\left(\mathrm{k}_{K} v_{i}\right)=$ $d(L)$, whence $1=(k+2) d(L), d(L)=1 /(k+2)=e(L)$.

Now suppose $d(L)=e(L)$ for all $L$ having $\leq j$ maximal simplices. Let $L$ have $j+1$ maximal simplices and let $v_{1}, \ldots, v_{r}$ denote the vertices of $L$. Consider $K=v * L \cong c L$ where $v$ is a vertex distinct from $v_{1}, \ldots, v_{r} . L=1 \mathrm{k}_{K} v$. Consider $\mathrm{lk}_{K} v_{i}$. This is clearly $v * \mathrm{lk}_{L} v_{i} \cong c \mathrm{k}_{L} v_{i}$. Thus $1 \mathrm{k}_{K} v_{i}$ is isomorphic to $\operatorname{st}_{L} v_{i}$. However, $\mathrm{st}_{L} v_{i}$ is clearly the union of maximal simplices of $L$. Hence $\mathrm{lk}_{K} v_{i}$ has $\leq j+1$ maximal simplices.

Now because $K$ is contractible, $1=\chi(K)=d(L)+\sum_{i=1}^{r} d\left(\mathrm{lk}_{K} v_{i}\right)$. Segregate the $v_{i}$ into two classes, $u_{i}, i=1, \ldots, s$, and $w_{i}, i=1, \ldots, t$, with $s+t=r$, by the criterion that $\mathrm{lk}_{K} u_{i}$ has $\leq j$ maximal simplices whereas $\operatorname{lk}_{K} w_{i}$ has $j+1$ maximal 
simplices. Thus $d\left(\mathrm{lk}_{K} u_{i}\right)=e\left(\mathrm{lk}_{K} u_{i}\right)$ for $i=1, \ldots, s$. Thus since

$$
\begin{aligned}
\chi(K) & =d(L)+\sum_{i=1}^{s} d\left(\mathrm{lk}_{K} u_{i}\right)+\sum_{i=1}^{t} d\left(\mathrm{lk}_{K} w_{i}\right) \\
& =e(L)+\sum_{i=1}^{s} e\left(\mathrm{lk}_{K} u_{i}\right)+\sum_{i=1}^{t} e\left(\mathrm{lk}_{K} w_{i}\right)
\end{aligned}
$$

we have

$$
d(L)+\sum_{i=1}^{t} d\left(\mathrm{lk}_{K} w_{i}\right)=e(L)+\sum_{i=1}^{t} e\left(\mathrm{lk}_{K} w_{i}\right)
$$

Note that $v$ and $w_{i}, i=1, \ldots, t$, may be characterized as those vertices of $K$ which are common to all the maximal simplices of $K$. It follows that $v, w_{1}, \ldots, w_{t}$ are the vertices of $\tau=\bigcap_{\alpha} \sigma_{\alpha}$, where $\left\{\sigma_{\alpha}\right\}$ is the set of maximal simplices of $K$. For any pair of these vertices, it is clear that there is thus a simplicial automorphism of $K$ carrying the first into the second. In other words, $L=1 \mathrm{k}_{K} v \cong \mathrm{k}_{K} w_{i}, i=1, \ldots, t$. Hence we see that $(t+1) d(L)=(t+1) e(L)$ whence $d(L)=e(L)$. This completes the proof.

\section{References}

[C] Cheeger, J., Spectual geometry of singular Riemannian spaces, J. Differential Geom. 18 (1983), 575-657.

[GM] Gelfand, 1. M., and MacPherson, R., A combinatorial formula for the Pontrjagin classes (preprint).

[K] Klee, V., A combinatorial analogue of Poincaré's duality theorem, Canad. J. Math. 16 (1964), 517-531.

[L] Levitt, N., Grassmannians and Gauss Maps in Piecewise-Linear Topology, Lecture Notes in Mathematics, Vol. 1366, Springer-Verlag, Berlin, 1989.

[LR] Levitt, N., and Rourke, C., The existence of combinatorial formulae for characteristic classes, Trans. Amer. Math. Soc. 239 (1978), 391-397.

[SCF] Steenrod, M., Cooke, G., and Finney R., Homology of Cell Complexes, Princeton University Press, Princeton, NJ, 1967.

[W1] Wall, C. T. C., Classification problems in differential topology, IV-Thickenings, Topology 5 (1966), 73-94.

[W2] Wall, C. T. C., Arithmetic invariants of subdivisions of complexes, Canad. J. Math. 18 (1966), 72-96.

Received September 13, 1989, and in revised form July 3, 1990 